\title{
Impact-parameter dependence of the electronic energy loss of fast ions
}

\author{
P. L. Grande \\ Instituto de Física da Universidade Federal do Rio Grande do Sul, Avenida Bento Goncalves 9500, 91501-970 Porto Alegre, Brazil \\ G. Schiwietz \\ Bereich F, Hahn-Meitner-Institut Berlin, Glienicker Strasse 100, D-14109 Berlin, Germany
}

(Received 6 May 1998)

\begin{abstract}
In this work we describe a model for the electronic energy loss of bare ions at high velocities. Starting from first-order perturbation theory we propose a simple formula to calculate the impact-parameter dependence of the electronic energy loss for all impact parameters. The physical inputs are the electron density and oscillators strengths of the atoms. A very good agreement is obtained with full first-order calculations.
\end{abstract}

[S1050-2947(98)01111-1]

PACS number(s): $34.50 . \mathrm{Bw}, 34.10 .+\mathrm{x}$

\section{INTRODUCTION}

The energy loss of light ions slowing down in matter is dominated by electronic processes at projectile velocities higher than the mean electron velocity. The basic mechanisms of the energy loss of a swift projectile are ionization and excitation of target atoms, well described in the framework of perturbation theory. The well known quadratic $Z$ (projectile charge) dependence of the electronic energy loss per unit path, the electronic stopping power, follows immediately from such a treatment [1]. Also, the Bethe formula [2] which yields the asymptotic high-energy limit of the stopping power may be derived directly from first-order perturbation theory.

Nowadays, besides the electronic stopping power, the impact-parameter-dependent energy loss is important due to an increasing number of experiments [3-7] and applications that require an accurate determination of the inelastic energy loss under channeling conditions. Although there exists a simple formula (the Bethe formula) for the integrated electronic stopping power at high energies, there is no corresponding solution, so far, for the electronic energy loss as a function of the impact parameter. In the literature, we may find only approximate analytical solutions using either the dipole expansion $[8,9]$, or the closure relation $[8,10]$, or solutions with the final states replaced by plane waves $[8,11]$. All of these models are valid for a restricted range of impact parameters.

Full calculations of the impact-parameter-dependent energy loss using first-order perturbation theory have been performed only recently [12] and a few years later the first coupled-channel calculations appeared $[13,14]$. These calculations are time consuming and results are only available for a few selected collision systems. Therefore, the computersimulation community has preferred to use semiempirical formulas [15] or other greatly simplified models $[16,17]$ in order to deal with inelastic collisions.

In this work we propose a model for the calculation of the impact-parameter-dependent energy loss at high projectile velocities, which reproduces full first-order calculations for all impact parameters without large-scale calculations. The physical inputs are the electronic density and the oscillator strengths for each allowed optical transition. If not indicated otherwise, atomic units $\left(e=1, m_{e}=1, \hbar=1\right)$ are used throughout the paper.

\section{MODEL}

According to the impact-parameter method [18] the electronic energy loss at high projectile energies can be obtained from first-order perturbation theory using straight line trajectories for the impinging ions. The energy loss due to capture and loss processes is of minor importance at high velocities and will be not considered in what follows. In order to calculate the energy loss due to target ionization and excitation we have to consider the amplitudes for each electronic transition from the ground-state $|0\rangle$ with energy $\varepsilon_{0}$ to a final state $|f\rangle$

$$
a_{f}(\vec{b})=-i \int_{-\infty}^{\infty} d t e^{i\left(\epsilon_{f}-\epsilon_{0}\right) t}\langle f|V(\vec{r}-\vec{R}(t))| 0\rangle
$$

Here $V(\vec{r}-\vec{R}(t))$ is the Coulomb interaction potential between the bare projectile ion with nuclear charge $Z$ and a target electron. Except for extraordinary small impact parameters, the ion trajectories are generally well described by straight lines $R(t)=\vec{v} t+\vec{b}$. As shown in Fig. $1, v$ is the projectile velocity and $b$ is the impact parameter vector of the collision. Each final bound or continuum state $\mathrm{f}$ can be defined by angular momentum quantum numbers $l_{f}, m_{f}$ and energy $\boldsymbol{\epsilon}_{f}$.

The electronic energy loss is calculated from the expression

$$
Q(b)=\sum_{f}\left|a_{f}(\vec{b})\right|^{2}\left(\epsilon_{f}-\epsilon_{0}\right)
$$

which involves a sum over all final target states and the corresponding computation of transition amplitudes. Usually this demands an computational effort that precludes the use of Eqs. (1) and (2) in a computer simulation code. Therefore, 


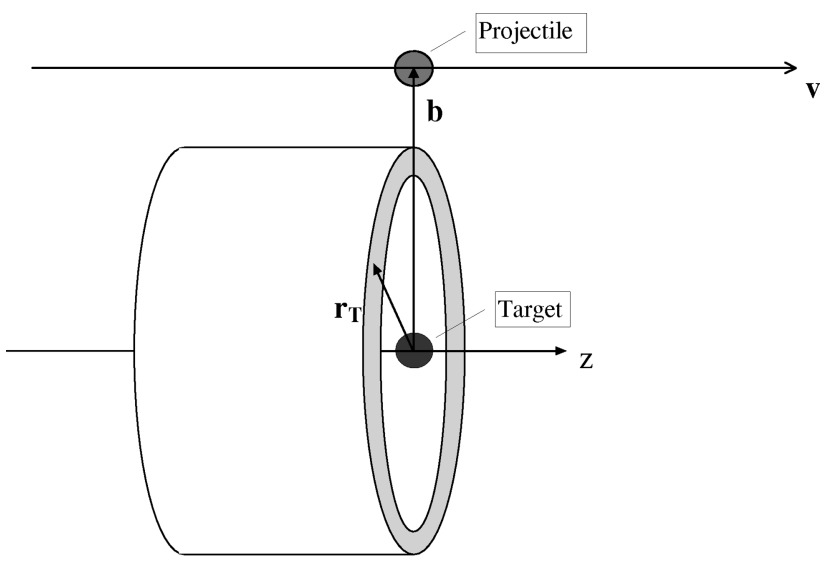

FIG. 1. Schematic representation of the collision geometry, showing the target and the projectile nucleus, the impact-parameter vector $\mathbf{b}$ and the projectile velocity $\mathbf{v}$. The cylinder represents the integrated electronic density along the ion flight direction.

we search for an approximate solution of both equations without being a large-scale calculation.

In the literature we may find many studies addressing the same problem [8-11]. They propose different approximations, all of them being valid in a limited range of impact parameters. At large impact parameters the so-called dipole approximation for $V(\vec{r}-\vec{R}(t))$ is used, where

$$
V(\vec{r}-\vec{R}(t))=-\frac{Z}{|\vec{r}-\vec{R}(t)|} \approx-\frac{Z}{R(t)}-\vec{r} \cdot \frac{\vec{R}(r) Z}{R^{3}(t)},
$$

and thus, an analytical expression $[9,19]$ for $Q(b)$ has been obtained:

$$
Q(b)=\frac{2 Z^{2}}{v^{2} b^{2}} \times \sum_{i} f_{i} g\left(\frac{\omega_{i} b}{v}\right)=T(b) \times \sum_{i} f_{i} g\left(\frac{\omega_{i} b}{v}\right)
$$

with $T(b)=2 Z^{2} /\left(v^{2} b^{2}\right)$ and

$$
g(x)=x^{2}\left(K_{0}^{2}(x)+K_{1}^{2}(x)\right) .
$$

$K_{0}$ and $K_{1}$ are the modified Bessel functions, $\omega_{i}$ are the transition energies $\left(\omega_{i} \equiv \epsilon_{i}-\epsilon_{0}\right)$, and $f_{i}$ are the well-known dipole-oscillator strengths $\left[f_{i}=2|\langle i|z| 0\rangle|^{2}\left(\epsilon_{i}-\epsilon_{0}\right)\right]$, which fulfill the sum rule $\Sigma_{i} f_{i}=1$ [1]. The function $g(\omega b / v)$ is displayed as dashed curve in Fig. 2. With increasing $\omega b / v$ it increases slightly from the value of 1 to a shallow maximum at $\omega b / v=0.17$ and at larger parameter values the function approaches zero exponentially. The solution given by Eq. (4) is exact for asymptotically large values of $b$, but it is completely inadequate for small impact parameters, where other multipole terms gain importance. In fact, Eq. (4) diverges for $b \rightarrow 0$. This follows from $\sum_{i} f_{i} g\left(\omega_{i} b / v\right)$ in Eq. (4) which approaches 1 in the limit $b \rightarrow 0$. As a consequence $Q(b)$ for $b \rightarrow 0$ turns out to be equal to the (divergent) classical energy transfer to an electron at rest, when the projectile velocity or the maximum energy transfer approaches infinity (in the socalled sudden approximation).

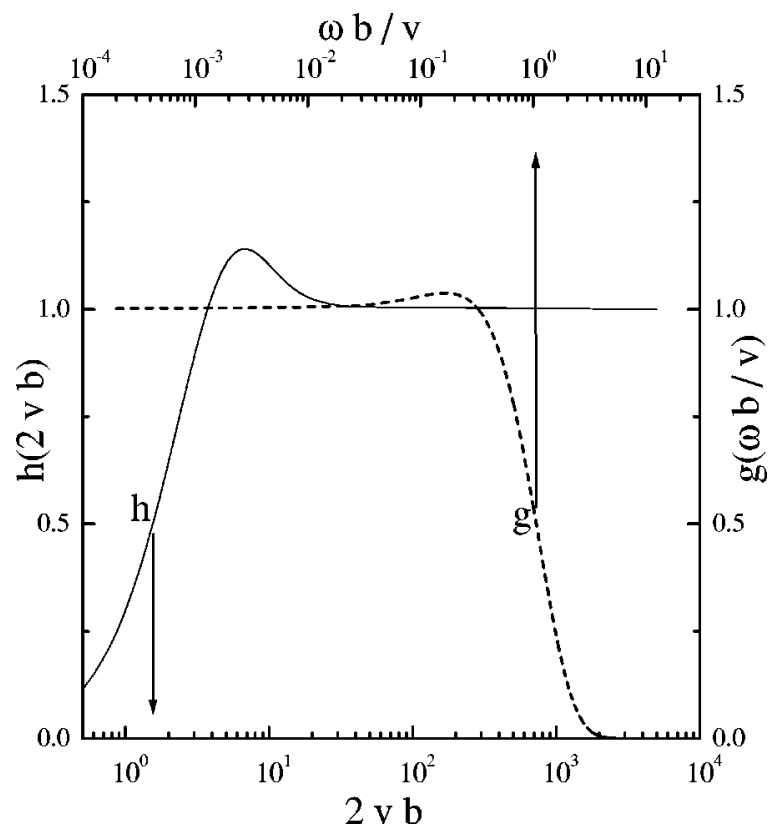

FIG. 2. The functions $g(x)$ and $h(x)$ from Eqs. (5) and (11) that describe the dipole terms and quasifree collisions, respectively. At high ion velocities the flat parts of both functions have a considerable overlap.

For intermediate impact parameters $b$ (somewhat larger than the shell radius, $\left.r_{\text {shell }}\right)$ and at high velocities the exponential factor in Eq. (1) can be neglected, when the collision time $\tau=r_{\text {shell }} / v$ is small compared to $1 / \omega_{i}$. Then, it is possible to sum over all final states analytically [8] by using the closure relation to obtain

$$
Q(b)=\int d^{2} r_{T} T\left(\vec{b}-\vec{r}_{T}\right) \int d z \rho\left(\vec{r}_{T}, z\right)
$$

with $T(b)=2 Z^{2} /\left(v^{2} b^{2}\right)$, which corresponds to the classical energy transfer to an electron at rest in the sudden approximation [19]. As shown in Fig. 1, the vector $\vec{r}_{T}$ is perpendicular to $z$, the direction of the projectile motion. $\int d z \rho\left(\vec{r}_{T}, z\right)$ is the electronic density integrated along the ion path (the cylinder in Fig. 1). Therefore, Eq. (6) agrees with the classical sudden-approximation expression of the energy loss for electrons at rest, distributed according to the coordinate-space density $\rho\left(\vec{r}_{T}, z\right)$. At small impact parameters, however, energy transfers as high as $\omega_{i}=2 v^{2}$ gain importance and a proper treatment of the two-body collision kinematics becomes necessary.

The influence of the target potential can be neglected for small impact parameters at high projectile energies [8]. In this case an analytical formula for $Q(b)$ can also be obtained by replacing the final target-continuum states by plane waves. The momentum distribution of the bound electron as well as the correct kinematics are considered in this case, so that the result should be similar to the classical binaryencounter approximation [20]. By following this procedure the electronic energy loss reads 


$$
\begin{aligned}
Q(b)= & \int d^{3} k \frac{k^{2}}{2}\left|a_{\vec{k}}(\vec{b})\right|^{2}=\int d^{3} k \frac{k^{2}}{2} \int_{-\infty}^{\infty} d t_{1} d t_{2} e^{i\left(k^{2} / 2\right)\left(t_{1}-t_{2}\right)} \\
& \times \int d^{3} r_{1} d^{3} r_{2} \phi_{0}^{*}\left(\vec{r}_{1}\right) \phi_{0}\left(\vec{r}_{2}\right) V\left(\vec{r}_{1}-\vec{R}\left(t_{1}\right)\right) \\
& \times V\left(\vec{r}_{2}-\vec{R}\left(t_{2}\right)\right) \frac{1}{(2 \pi)^{3}} e^{i \vec{k}\left(\vec{r}_{1}-\vec{r}_{2}\right)}
\end{aligned}
$$

An integration over the orientation of $\vec{k}$ leads to the following integral:

$$
\int d \Omega_{k} e^{i \vec{k} \cdot\left(\vec{r}_{1}-\vec{r}_{2}\right)}=4 \pi \frac{\sin \left(k\left|\vec{r}_{1}-\vec{r}_{2}\right|\right)}{k\left|\vec{r}_{1}-\vec{r}_{2}\right|}
$$

which deviates significantly from zero only for the very narrow range $\left|\vec{r}_{1}-\vec{r}_{2}\right|<1 / k$. Since at high velocities large values of $k$ dominate the integral, the electronic ground-state wave function $\phi_{0}\left(\vec{r}_{2}\right)$ can be replaced by $\phi_{0}\left(\vec{r}_{1}\right)$. However, this peaking approximation removes the exact treatment of the momentum distribution of the bound electron. Without any further approximations the energy loss from Eq. (7) can be written as

$$
Q(b)=\int d^{2} r_{T} \mathcal{T}\left(\vec{b}-\vec{r}_{T}\right) \int d z \rho\left(\vec{r}_{T}, z\right)
$$

with

$$
\mathcal{T}(b)=\frac{2 Z^{2}}{v^{2} b^{2}} \times h(2 v b)=T(b) \times h(2 v b),
$$

and

$$
h(x)=\frac{x^{2}}{2} \int_{0}^{1} d y y K_{0}\left(x y^{2}\right) J_{0}\left(x y \sqrt{1-y^{2}}\right) .
$$

A similar expression was also derived in Ref. [11] but involving Bessel functions of higher order. The function $h(2 v b)$ approaches zero for $b \ll 1 / v$ and it reaches 1 for large values of $b$. In the latter case, the energy transfer resembles again the classical energy transfer to a statistical distribution of electrons at rest. It is pointed out that the results from Eq. (10) are target independent.

In what follows we propose a general formula that interpolates between all approximations described above and is applicable for all impact parameters, namely,

$$
Q(b)=\int d^{2} r_{T} \mathcal{K}\left(\vec{b}-\vec{r}_{T}\right) \int d z \rho\left(\vec{r}_{T}, z\right)
$$

with

$$
\mathcal{K}(b)=\frac{2 Z^{2}}{v^{2} b^{2}} \times h(2 v b) \times \sum_{i} f_{i} g\left(\frac{\omega_{i} b}{v}\right),
$$

This function joins smoothly all three regions of impact parameters described above. The first two terms in Eq. (13) describe violent binary collisions and the last term accounts for the long-range dipole transitions. The first integral $\int d^{2} r_{T} \ldots$ in Eq. (12) describes a convolution with the initial electron density also outside the projectile path and yields nonlocal contributions to the energy loss.

In the following it will be shown that Eqs. (12) and (13) are consistent with the above-discussed three limiting impact parameter behaviors as well as with the Bethe formula. For large impact parameters $\left(\mathrm{b} \gg \mathrm{r}_{\text {shell }}\right)$ the convolution from Eq. (12) can be separated using a peaking approximation as

$$
\begin{aligned}
& \int d^{2} r_{T} \mathcal{K}\left(\vec{b}-\vec{r}_{T}\right) \int d z \rho\left(\vec{r}_{T}, z\right) \\
& \approx \int d^{2} r_{T} \mathcal{K}(\vec{b}) \int d z \rho\left(\vec{r}_{T}, z\right)=\mathcal{K}(\vec{b}),
\end{aligned}
$$

since the range of perpendicular distances $r_{T}$, where the electron density is significant, is small compared to $b$ and the function $\mathcal{K}\left(\vec{b}-\vec{r}_{T}\right)$ is not sensitive to small variation of $r_{T}$ at high velocities. Hence, Eq. (12) agrees with the results obtained from the dipole approximation given by Eq. (4), because $h(2 v b)$ approaches 1 for large impact parameters (see Fig. 2).

On the other hand, the model from Eq. (13) reproduces the small-impact-parameter limit described by Eq. (10) since $\sum_{i} f_{i} g\left(\omega_{i} b / v\right)$ tends to 1 for $b \rightarrow 0$ (see Fig. 2). Finally, according to Fig. 2, the higher the projectile velocity $v$ the better the separation of the limits of small and large impact parameters, where the functions $g$ and $h$ approach zero. Consequently, the flat impact-parameter regions of both functions merge into each other at intermediate impact parameters [where both functions $\Sigma_{i} f_{i} g\left(\omega_{i} b / v\right)$ and $h(2 v b)$ approach unity]. Therefore, $\mathcal{K}(b)$ approaches $T(b)$ from Eq. (6) and the proposed energy-loss function incorporates all the above-discussed approximations in their restricted impact-parameter regimes.

At high projectile velocities $v^{2} \gg \omega_{i}$, there exists an impact parameter $b_{c}$ (in general even an impact-parameter range) for which both functions $h$ and $g$ are nearly equal to 1 . Then, the integrated electronic stopping cross section $S_{e}$ can be calculated from

$$
\begin{aligned}
S_{e} & =\int d^{2} b Q(b)=2 \pi N \int_{0}^{\infty} d b b \mathcal{K}(b) \\
& \approx \frac{4 \pi N Z^{2}}{v^{2}}\left[\int_{0}^{b_{c}} d b h(2 v b) / b+\int_{b_{c}}^{\infty} d b \sum_{i} f_{i} g\left(\frac{\omega_{i} b}{v}\right) / b\right] \\
& =\frac{4 \pi N Z^{2}}{v^{2}}\left[\ln \left(\frac{2 v b_{c}}{1.123}\right)+\sum_{i} f_{i} \ln \left(\frac{1.123 v}{\omega_{i} b_{c}}\right)\right]
\end{aligned}
$$

$$
=\frac{4 \pi N Z^{2}}{v^{2}} \ln \left(\frac{2 v^{2}}{I_{\text {Bethe }}}\right)
$$

with

$$
\ln \left(I_{\text {Bethe }}\right)=\sum_{i} f_{i} \ln \left(\omega_{i}\right)
$$


and $N$ is the total number of electrons. Thus, the integration of Eq. (12) over all impact parameters provides the electronic stopping cross section according to the Bethe formula [2]. Therefore, for asymptotically high projectile energies the present model gives the exact limiting impact-parameter dependences of the electronic energy loss.

For use in practical applications the functions $h(x)$ and $g(x)$ can be expressed in terms of elementary functions fitted to the numerical results,

$$
h(x)=\frac{x^{2}}{x^{2}+\alpha^{2}}+\frac{\beta x^{2}}{1+\gamma x^{4}}
$$

with $\alpha=1.551, \beta=0.0123, \gamma=0.0009$, and

$$
g(x)=(1-0.174 \sqrt{x}+\pi x) \exp (-2 x),
$$

which yield negligible uncertainties in Eq. (12) for $0 \leqslant x$ $<\infty$

It is pointed out that the formulas for $\mathrm{Q}(b)$ as given above are strictly valid only for a one-electron system. In the framework of the independent-particle model, however, we can still use the results of Eq. (12), but we have to consider the electronic density and the oscillator strengths for each electron of all occupied target shells.

\section{DISCUSSIONS AND CONCLUSIONS}

In Figs. 3-5 we show the results of the present model for the impact-parameter dependence of the mean energy loss of bare ions colliding with $\mathrm{H}, \mathrm{He}$, and $\mathrm{Ne}$ targets (dashed lines) in comparison with full first-order calculations [semiclassical approximation (SCA)] [22] (solid lines). The electronic densities were obtained from the analytical formula for the $\mathrm{H}$ target and from Hartree-Fock-Slater densities for $\mathrm{He}$ and $\mathrm{Ne}$ $[21,22]$. The oscillators strengths are taken from Ref. [23] for $\mathrm{H}$ and from Ref. [22] for the He target.

For collisions with hydrogen (see Fig. 3) we have considered two initial states, namely, $\mathrm{H}(1 s)$ and $\mathrm{H}(2 s)$. All curves are labeled with the corresponding ion energy. In all six cases we obtain a very good agreement with full SCA calculations. Even the influence of the node structure of the $2 s$ wave function is very well described by the present model. As we have shown in earlier $[13,14]$, first-order perturbation theory (SCA) yields reliable results for protons at energies above $150 \mathrm{keV}$. For heavier ions the projectile velocity should be high enough $(Z / v<0.8$ for the hydrogen ground state) to fulfill the condition of a small perturbation.

For a quick estimate of the electronic energy loss as a function of the impact parameter we may replace the sets of oscillator strengths $\left\{f_{i}\right\}$ and transition energies $\left\{\omega_{i}\right\}$ by just one oscillator strength $f=1$ corresponding to a mean energy transfer $\bar{\omega}$. Since small energy transfers are dominant at large impact parameters, the use of a single oscillator strength will underestimate the energy loss at asymptotically large impact parameters. The result of this approximation, represented by a dotted line, is shown in Fig. 4 for a He target excited from the ground-state by a bare projectile at $300 \mathrm{keV} / \mathrm{u}$. Here we have used $\bar{\omega}=\exp \left[\Sigma_{i} f_{i} \ln \left(\omega_{i}\right)\right]=39.3 \mathrm{eV}$

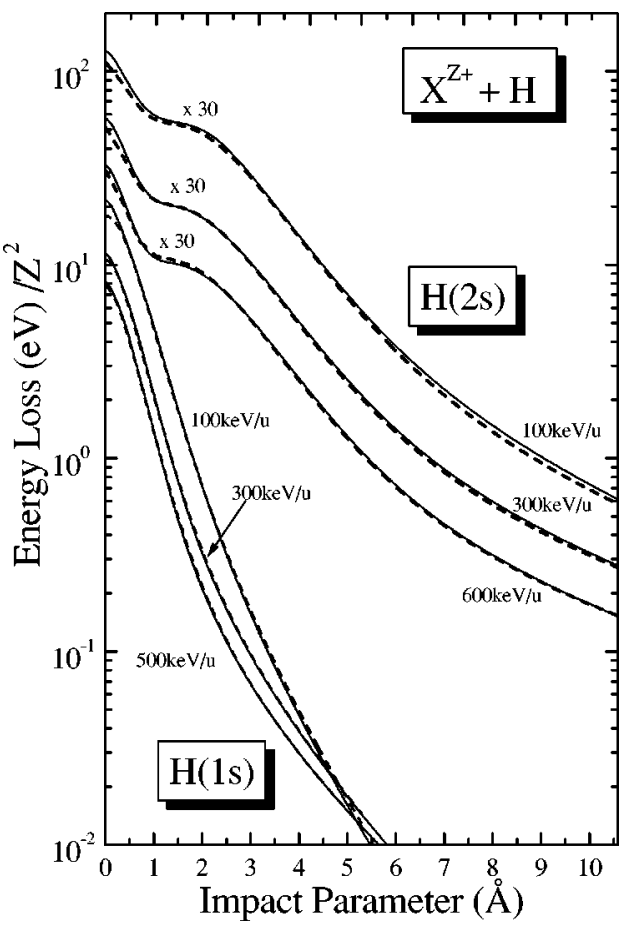

FIG. 3. The electronic energy loss is displayed as a function of the impact parameter for bare ions (with nuclear charge equal to $Z$ ) colliding with $\mathrm{H}$ atoms. Results are shown for scaled projectile energies of 100, 300, 500, and $600 \mathrm{keV}$ per atomic mass unit (u) and for the $1 s$ ground state as well as for the $2 s$ initial state (these curves are multiplied by a factor of 30). The solid lines represent full first-order calculations from Ref. [22] and the dashed lines correspond to the present model.

with $f_{i}$ and $\omega_{i}$ obtained from Ref. [22]. From this figure we can observe a good agreement with full SCA calculations for intermediate and small impact parameters. Only for large impact parameters the use of the exact set of oscillators strengths and $\omega_{i}$ turns out to be important. At small impact parameters all results are in good agreement with an experimental data point from Ref. [24] $(45 \pm 6 \mathrm{eV})$, which was derived for an impact parameter of 0.02 a.u. from differential ionization, electron-capture and excitation probabilities.

Finally, Fig. 5 shows a comparison for Ne that has three subshells and therefore the electronic density for each subshell was taken into account. Here, only a single oscillator strength and transition energy was considered for each subshell and the mean transfer energy $\bar{\omega}_{s}$ was assumed to be equal to $\alpha I_{s}$, with $I_{s}$ being the binding energy of the shell $s$ under consideration and

$$
\alpha=I_{\text {Bethe }} / \exp \left(\frac{1}{N} \sum_{s} n_{s} \ln \left(I_{s}\right)\right),
$$

where $n_{s}$ is the number of electrons of the shell $s$. For Ne the Hartree-Fock-Slater [21] values of $I_{s}$ were used $\left(I_{1 s}\right.$ $=857 \mathrm{eV}, I_{2 s}=43 \mathrm{eV}$, and $I_{2 p}=20 \mathrm{eV}$ ). This choice is consistent with the mean energy transfer $I_{B e t h e}$ from the Bethe formula [see Eqs. (15) and (16)]. For heavy targets the value of $I_{B e t h e}$ is proportional to the atomic number of the target atom $\left(Z_{\text {target }}\right)$, i.e., $I_{\text {Bethe }} \approx 12 e V^{*} Z_{\text {target }}[25,26]$. 


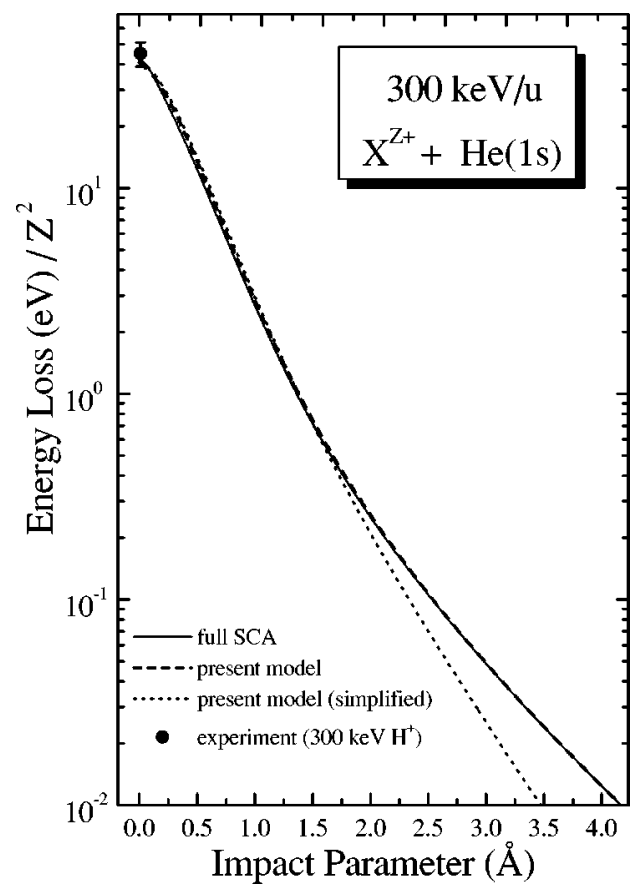

FIG. 4. The electronic energy loss is displayed as a function of the impact parameter for bare ions (with nuclear charge equal to $Z$ ) colliding with He atoms. Results are shown for a scaled projectile energy of $300 \mathrm{keV}$ per atomic mass unit $(\mathrm{u})$ and the target $1 \mathrm{~s}$ ground state. The solid line represents full first-order calculations from Ref. [22] and the dashed line corresponds to the present model. The dotted line is also computed using the present model, but the sum over oscillator strengths $\Sigma_{i} f_{i} g\left(\omega_{i} b / v\right)$ in Eq. (13) has been replaced by a single function $g(\bar{\omega} b / v)$ with $\bar{\omega}=39.3 \mathrm{eV}$. The experimental data point at $b=0.02$ a.u. stems from triplecoincidence measurements [24].

The agreement between the present model using $\bar{\omega}_{s}$ $=2.43 * I_{s}$ (dotted line) and the full first-order calculations (solid lines) is worse than for the $\mathrm{H}$ and $\mathrm{He}$ targets but still reasonable concerning the total electronic energy loss (the discrepancy is about $15 \%$ ). A common feature of our simplified model results is the underestimated energy loss at large impact parameters as discussed above. The largest discrepancy, however, is observed for excitation of the Ne $2 s$ shell. In this case the node structure of the $2 s$ wave function is known to lead to destructive interferences that reduce the ionization probabilities. Since the use of a single oscillator strength cannot account for this interference, our simplified model results overestimate the SCA results by $30-40 \%$ for the $2 s$ shell.

Furthermore, it should be noted that the full SCA calculations account for the Pauli exclusion principle. This means that transition from the $2 s$ to the $2 p$ state or from $1 s$ to $2 s$ or $2 p$ states are explicitly excluded. The Pauli principle can be taken into account by our present model [Eq. (12)] if we use the exact sets of oscillator strengths, but it is clearly not accounted for by the simplified single-oscillator model. The energy loss due to $K$-shell ionization should be most sensitive to this blocking effect due to the Pauli exclusion principle and our model results for the $K$ shell overestimate the SCA results by only about $20 \%$ at zero impact parameter. In principle, the mean energy transfer may be chosen to account

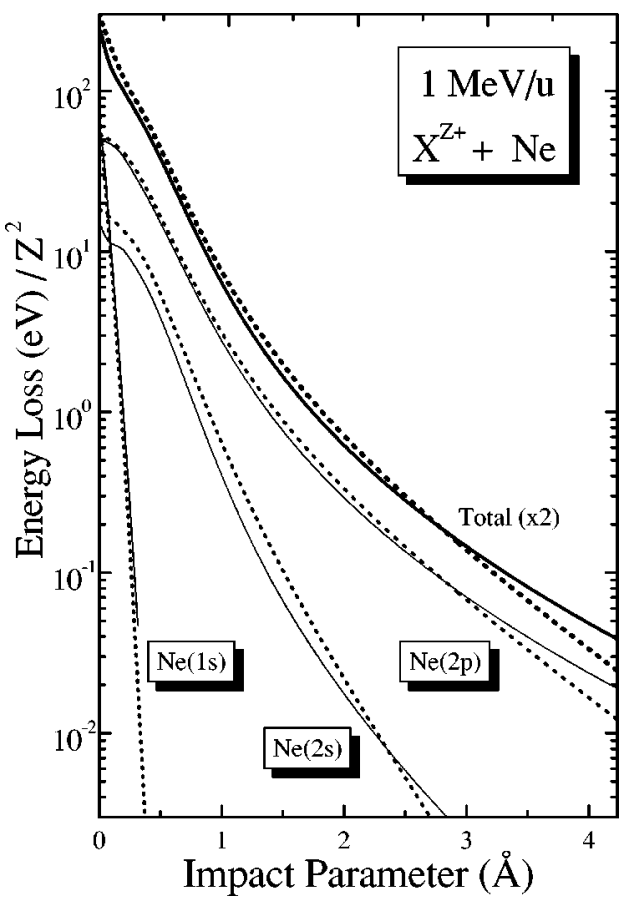

FIG. 5. The electronic energy loss is displayed as a function of the impact parameter for bare ions (with nuclear charge equal to $Z$ ) colliding with $\mathrm{Ne}$ atoms. Results are shown for a scaled projectile energy of $1 \mathrm{MeV}$ per atomic mass unit (u) and for the target shells $1 s, 2 s$, and $2 p$. The solid lines represent full first-order calculations from Ref. [22] and the dotted lines correspond to the present model, using a single function $g\left(\bar{\omega}_{s} b / v\right)$ for each shell $s$ with binding energy $I_{s}$, where the mean excitation energy $\bar{\omega}_{s}$ was simply replaced by $\bar{\omega}_{s}=2.43 * I_{s}$. The total energy loss, i.e., the sum over the shell contributions, is multiplied by a factor of 2 and displayed as thick solid and dotted curves.

also for the unphysical deexcitation processes into occupied states. Under this condition, the blocking effect due to the Pauli principle disappears for the total energy loss (sum over all subshells) in a first-order treatment since the sum over all forbidden transitions cancels out (the probability for a transition from $2 s$ to $2 p$ is equal to the reverse one, but with a transition energy of opposite sign).

In conclusion, we have developed a simple formula for the electronic energy loss as a function of the impact parameter that is valid at high energies and for a wide range of impact parameters. The integral over all impact parameters recovers the Bethe formula. The implementation of Eq. (12), for instance in simulation codes that describe channeled particles, is straightforward and depends basically on the electronic density and the mean transition energy. If uncertainties in excess of $10 \%$ are not tolerable, the model results may be brought in nearly perfect agreement with full SCA results by using tabulated oscillator strengths instead of only a single mean transition energy.

\section{ACKNOWLEDGMENTS}

This work was partially supported by Conselho Nacional de Desenvolvimento Científico e Tecnológico (CNPq), and by the Alexander-von-Humboldt foundation. 
[1] H. A. Bethe and R. W. Jackiw, Intermediate Quantum Mechanics, 2nd ed. (Benjamin, New York, 1968).

[2] H. Bethe, Ann. Phys. (Leipzig) 5, 325 (1930).

[3] A. Dygo, M. A. Boshart, L. E. Seiberling, and N. M. Kabachnik, Phys. Rev. A 50, 4979 (1995); A. A. Bailes III, M. A. Boshart, and L. E. Seiberling, Nucl. Instrum. Methods Phys. Res. B 136-138, 804 (1998).

[4] Alertazzi, M. Bianconi, G. Lulli, and R. Nipoli, Nucl. Instrum. Methods Phys. Res. B 118, 128 (1996).

[5] W. R. Wampler, Phys. Rev. B 51, 4998 (1995).

[6] A. La Ferla, E. Rimini, A. Carnera, A. Gasparotto, G. Ciavola, and G. Ferla, Radiat. Eff. 129, 133 (1994) and references therein.

[7] J. H. R. dos Santos, P. L. Grande, M. Behar, H. Boudinov, and G. Schiwietz, Phys. Rev. B 55, 4332 (1997).

[8] F. Bloch, Ann. Phys. (Leipzig) 16, 285 (1933).

[9] C. O. Reinhold and J. Burgdoerfer, J. Phys. B 26, 3101 (1993).

[10] K. Dettmann, Z. Phys. A 272, 227 (1975).

[11] V. A. Knodyrev and E. I. Sirotinin, Phys. Status Solidi B 116, 659 (1983).

[12] N. M. Kabachnik, V. N. Kondratev, and O. V. Chumanova, Phys. Status Solidi B 145, 103 (1988).

[13] G. Schiwietz, Phys. Rev. A 42, 296 (1990).

[14] G. Schiwietz and P. L. Grande, Nucl. Instrum. Methods Phys. Res. B 69, 10 (1992); P. L. Grande and G. Schiwietz, Phys. Rev. A 47, 1119 (1993); P. L. Grande and G. Schiwietz, Nucl. Instrum. Methods Phys. Res. B 132, 264 (1997).
[15] O. Oen and M. Robinson, Nucl. Instrum. Methods 132, 647 (1976).

[16] O. B. Firsov, Zh. Éksp. Teor; Fiz. 36, 1517 (1959) [Sov. Phys. JETP 9, 1076 (1959)].

[17] J. Lindhard and A. Winther, K. Dan. Vidensk. Selsk. Mat. Fys. Medd. 34, 4 (1964).

[18] J. Bang and J. M. Hansteen, K. Dan. Vidensk. Selsk. Mat. Fys. Medd. 31, 13 (1959); L. Wilets and S. J. Wallace, Phys. Rev. 169, 84 (1968); M. R. Flannery and K. J. MacCann, Phys. Rev. A 8, 2915 (1973).

[19] J. D. Jackson, Classical Electrodynamics (Wiley, New York, 1975), Chap. 13.

[20] L. Vriens, in Case Studies in Atomic Physics, edited by E. W. Mc. Daniel and M. R. C. Mc Dowell (North-Holland, Amsterdam, 1969), Vol. 1, pp. 335.

[21] F. Herman and S. Skillmann, in Atomic Structure Calculations (Prentice-Hall, Englewood Cliffs, NJ, 1963).

[22] The numerical procedures are same as used in Refs. $[13,14]$.

[23] M. Inokuti, Rev. Mod. Phys. 43, 297 (1971).

[24] B. Skogvall and G. Schiwietz, Phys. Rev. A 46, 5687 (1992); G. Schiwietz, B. Skogvall, N. Stolterfoht, D. Schneider, V. J. Montemayor, and H. Platten, Nucl. Instrum. Methods Phys. Res. B 40/41, 178 (1989).

[25] C. J. Bakker and E. Segre, Phys. Rev. 81, 489 (1951); D. C. Sachs and J. R. Richardson, ibid. 83, 834 (1951).

[26] H. H. Andersen and J. F. Ziegler, in Stopping Powers and Ranges in all Elements (Pergamon, New York, 1977). 